\title{
LA-UR-13-21570
}

Approved for public release; distribution is unlimited.

Title:

Author(s):

Intended for:

\section{Double Beta Decay}

\author{
Elliott, Steven R.
}

Review of underground facility and double-beta decay and dark matter experiments in Korea. I am a reviewer because of my expertise in double beta decay. I will present background material at a short symposium covering material for the review.

\section{- LosAlamos NATIONAL LABORATORY

\author{
EST. 1943
}

Disclaimer:

Los Alamos National Laboratory, an affirmative action/equal opportunity employer,is operated by the Los Alamos National

Security, LLC for the National NuclearSecurity Administration of the U.S. Department of Energy under contract DE-AC52-06NA25396.

By approving this article, the publisher recognizes that the U.S. Government retains nonexclusive, royalty-free license to

publish or reproduce the published form of this contribution, or to allow others to do so, for U.S. Government purposes.

Los Alamos National Laboratory requests that the publisher identify this article as work performed under the auspices of the

U.S. Departmentof Energy. Los Alamos National Laboratory strongly supports academic freedom and a researcher's right to publish; as an institution, however, the Laboratory does not endorse the viewpoint of a publication or guarantee its technical correctness. 


\section{Los Alamos}

Steve Elliott

\section{Double Beta Decay}

-Neutrinos

- Science of $\beta \beta$

-MAJORANA DEMONSTRATOR 


\section{Why Neutrinos?}

$v$ properties are critical input to many physics questions

- Particle/Nuclear Physics

- Fundamental questions about standard model

- Fundamental issues regarding interactions

- Cosmology

- Large scale structure

- Leptogenesis and matter-antimatter asymmetry

- Astrophysics

- Supernova explosions

- Solar burning 


\section{Neutrinos: What do we want to know?}

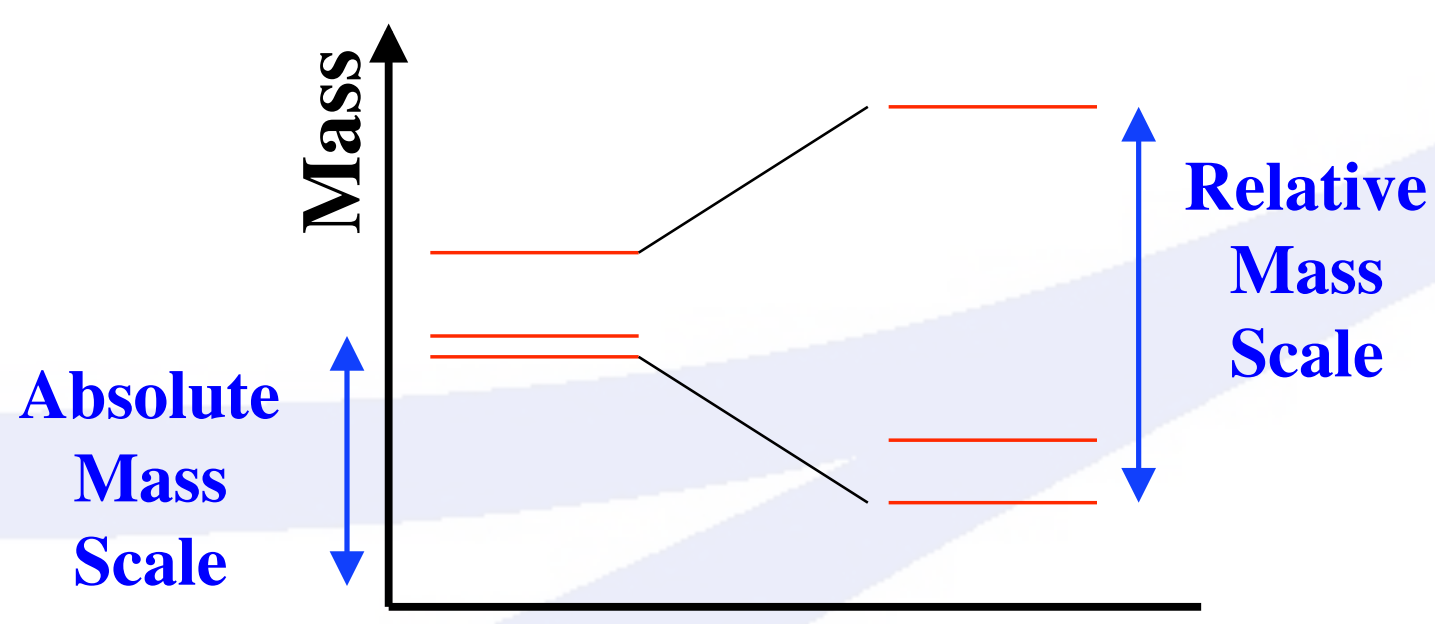

Dirac or Majorana

$$
\left(\begin{array}{l}
v_{\uparrow} \\
v_{\downarrow} \\
\bar{v}_{\downarrow} \\
\bar{v}_{\uparrow}
\end{array}\right) \text { or }\left(\begin{array}{l}
v_{\uparrow} \\
v_{\downarrow}
\end{array}\right)
$$

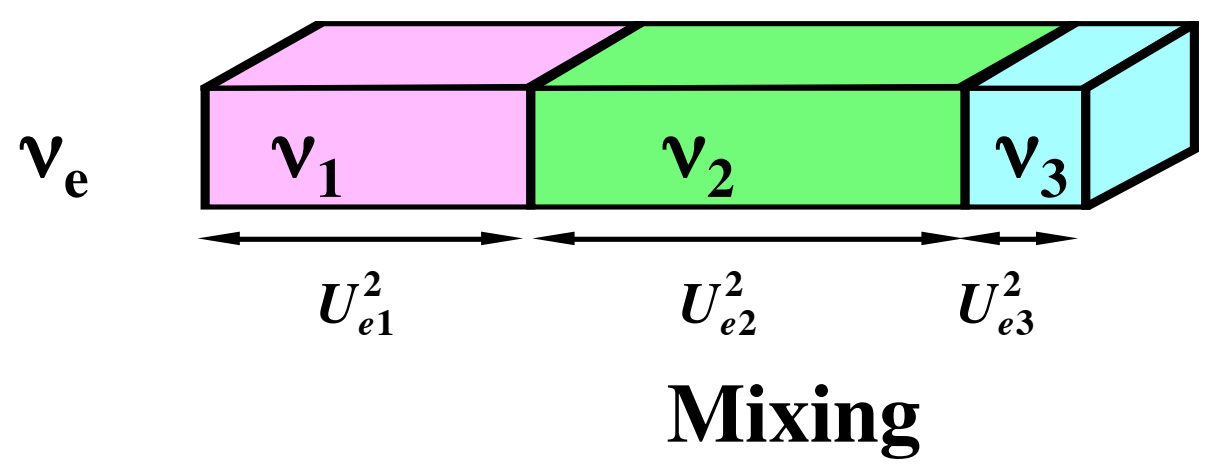




\section{Neutrino Masses: What do we know?}

- The results of oscillation experiments indicate $v$ do have mass!, set the relative mass scale, and a minimum for the absolute scale.

- $\beta$ decay experiments set a maximum for the absolute mass scale.

$$
50 \mathrm{meV}<\mathrm{m}_{v}<2200 \mathrm{meV}
$$




\section{We also know $v$ mix.}

The weak interaction produces $v_{e}, v_{\mu}, v_{\tau}$.

These are not pure mass states but a linear combination of mass states.

$$
\left(\begin{array}{c}
v_{e} \\
v_{\mu} \\
v_{\tau}
\end{array}\right)=\left(\begin{array}{lll}
U_{e 1} & U_{e 2} & U_{e 3} \\
U_{\mu 1} & U_{\mu 2} & U_{\mu 3} \\
U_{\tau 1} & U_{\tau 2} & U_{\tau 3}
\end{array}\right)\left(\begin{array}{c}
v_{1} \\
v_{2} \\
v_{3}
\end{array}\right)
$$

Oscillation experiments indicate that $v$ mix and constrain $U_{\alpha i}$. 


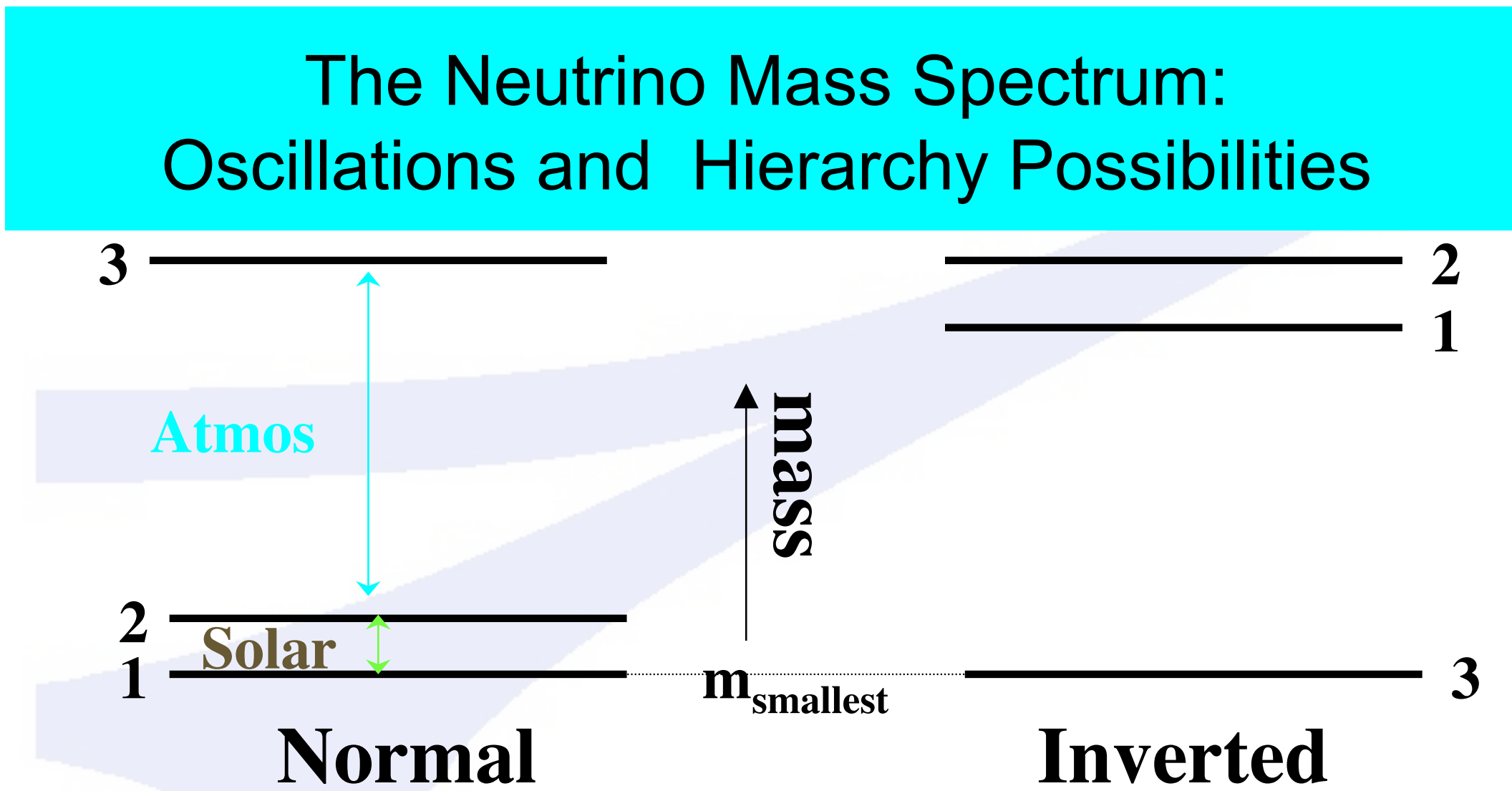

$v_{e}$ is composed of a large fraction of $v_{1}$. 


\section{Example $\beta \beta$ Decay Scheme}

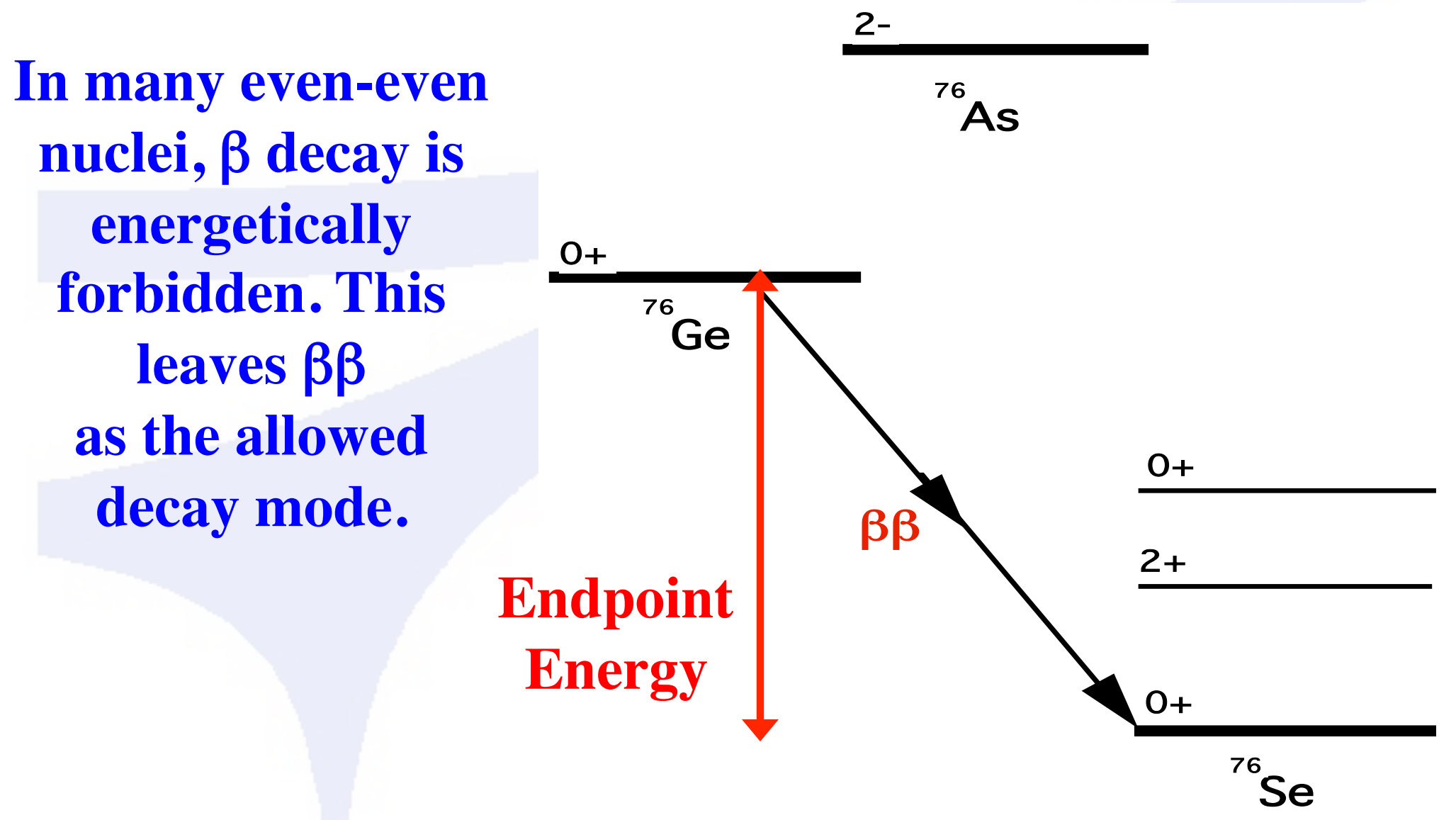




\section{What is $\beta \beta$ ?}
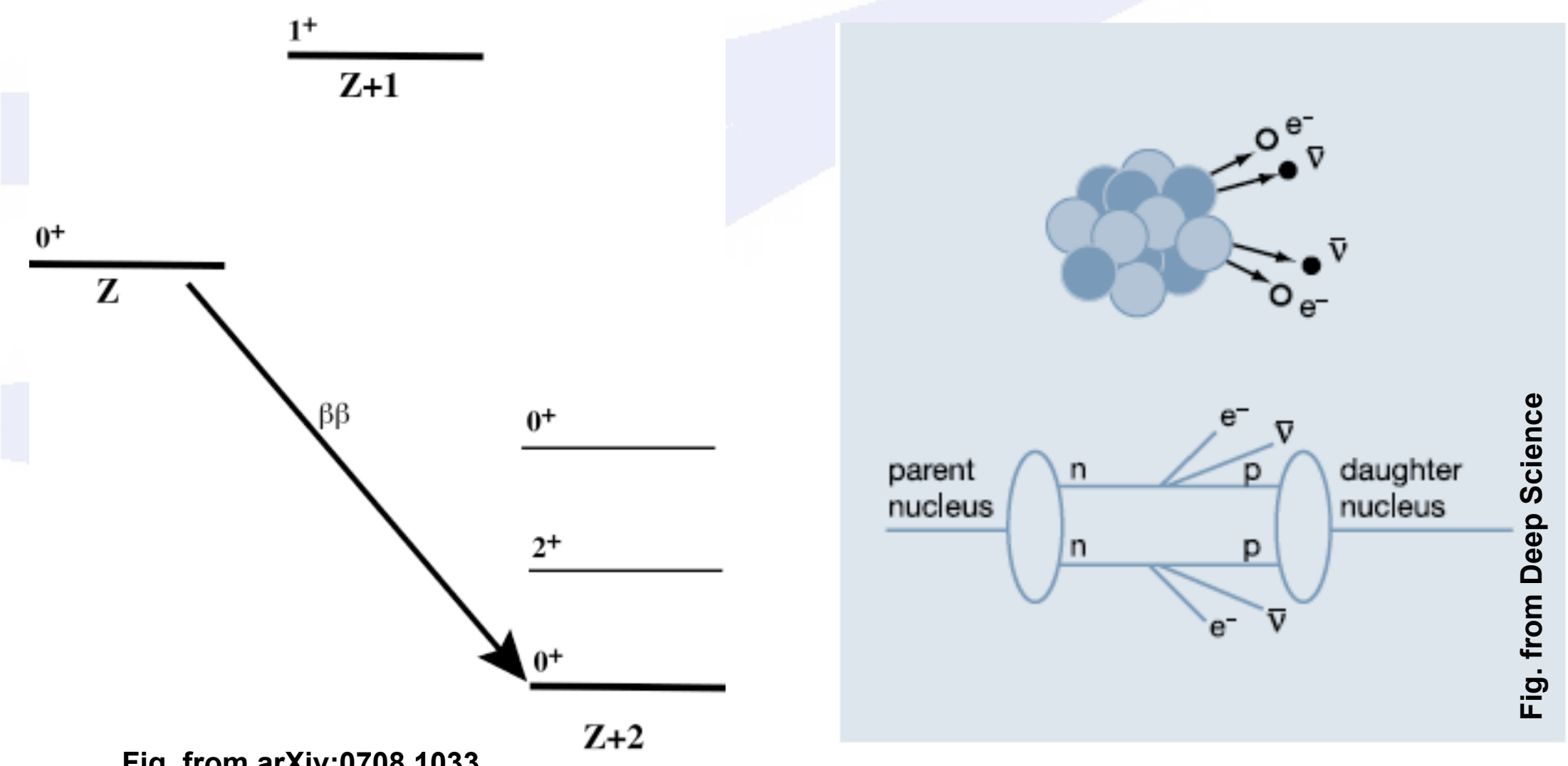

Fig. from arXiv:0708.1033 


\section{What is $\beta \beta$ ?}

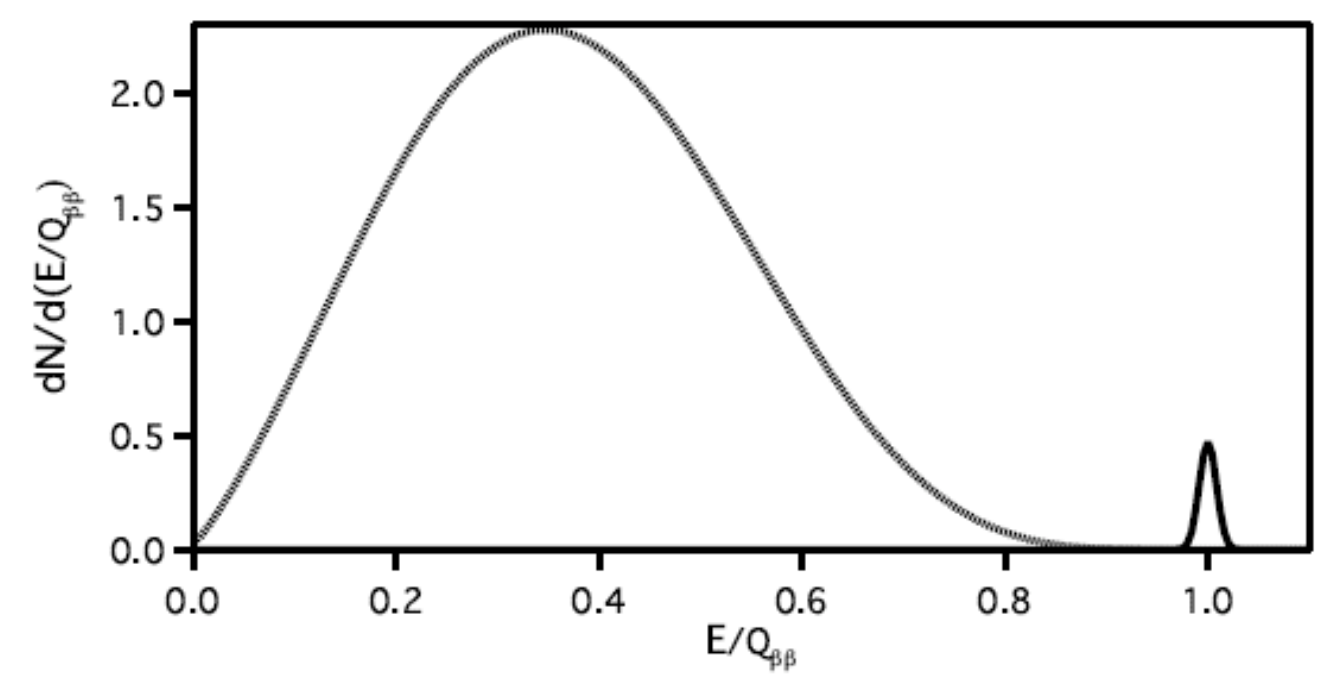

Fig. from arXiv:0708.1033

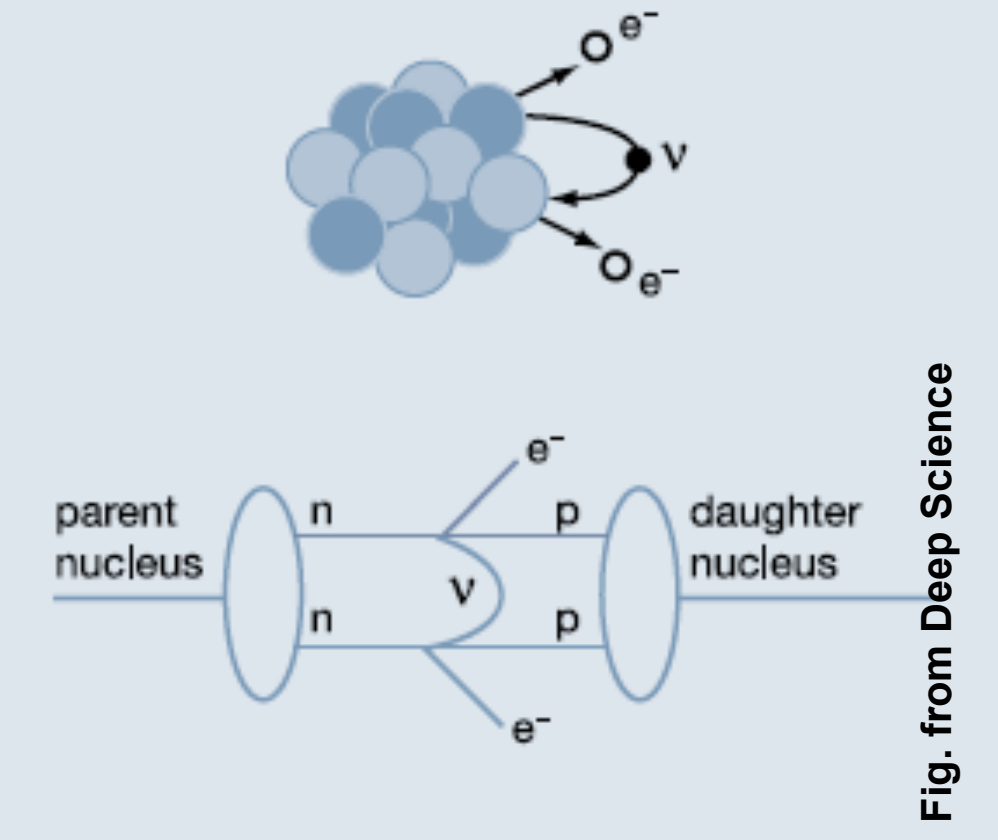

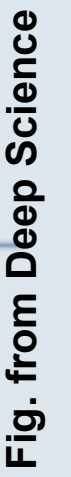




\section{$\beta \beta$ Decay Rates}

$$
\Gamma_{2 v}=G_{2 v}\left|M_{2 v}\right|^{2} \quad \Gamma_{0 v}=G_{0 v}\left|M_{0 v}\right|^{2} m_{v}^{2}
$$

G are calculable phase space factors.

$$
\mathbf{G}_{0 v} \sim \mathbf{Q}^{5}
$$

IMI are nuclear physics matrix elements.

Hard to calculate.

$\mathbf{m}_{v}$ is where the interesting physics lies. 


\section{What about mixing, $\mathrm{m}_{v} \& \beta \beta(0 v)$ ?}

$$
\text { No mixing: }\left\langle m_{\beta \beta}\right\rangle=m_{v_{e}}=m_{1}
$$

$$
\begin{aligned}
\left\langle m_{\beta \beta}\right\rangle & =\sum_{i=1}^{3}\left|U_{e i}\right|^{2} m_{i} \varepsilon_{i} \begin{array}{l}
\text { virtual } v \\
\text { exchange }
\end{array} \\
\varepsilon & = \pm 1, \mathrm{CP} \text { cons. }
\end{aligned}
$$

Compare to $\beta$ decay result:

$$
\left\langle m_{\beta}\right\rangle=\sqrt{\sum_{i=1}^{3}\left|U_{e i}\right|^{2} m_{i}^{2}}
$$
real $v$ emission
Compare to cosmology:

$$
\sum=\sum m_{i}
$$




\section{$\beta \beta$ Sensitivity}

(mixing parameters from arXiv:1106.6028)

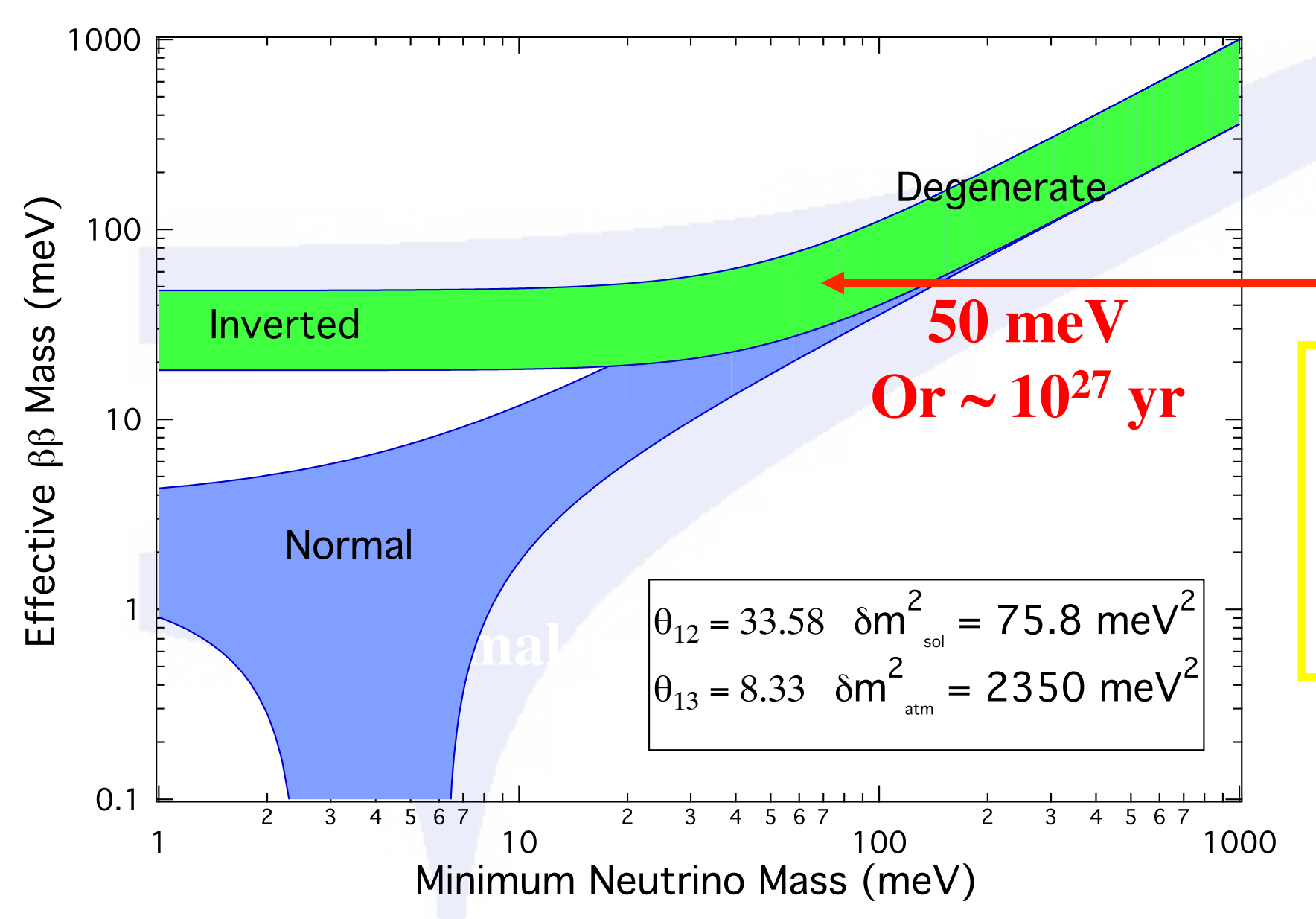

Even a null result will constrain the possible mass spectrum possibilities!

A $\mathrm{m}_{\beta \beta}$ limit of $\sim 20$ meV would exclude Majorana neutrinos in an inverted hierarchy. 


\section{$\beta \beta$ and the neutrino}

- $\beta \beta(0 v)$ decay rate proportional to neutrino mass

- Most sensitive technique (if Majorana particle)

- Decay can only occur if Lepton number conservation is violated

- Leptogenesis?

- Decay can only occur if neutrinos are massive Majorana particles

- Critical for understanding incorporation of mass into standard model

- $\beta \beta$ is only practical experimental technique to answer this question

- Fundamental nuclear/particle physics process 


\section{$\beta \beta$ Candidates}

\section{There are a lot of them!}

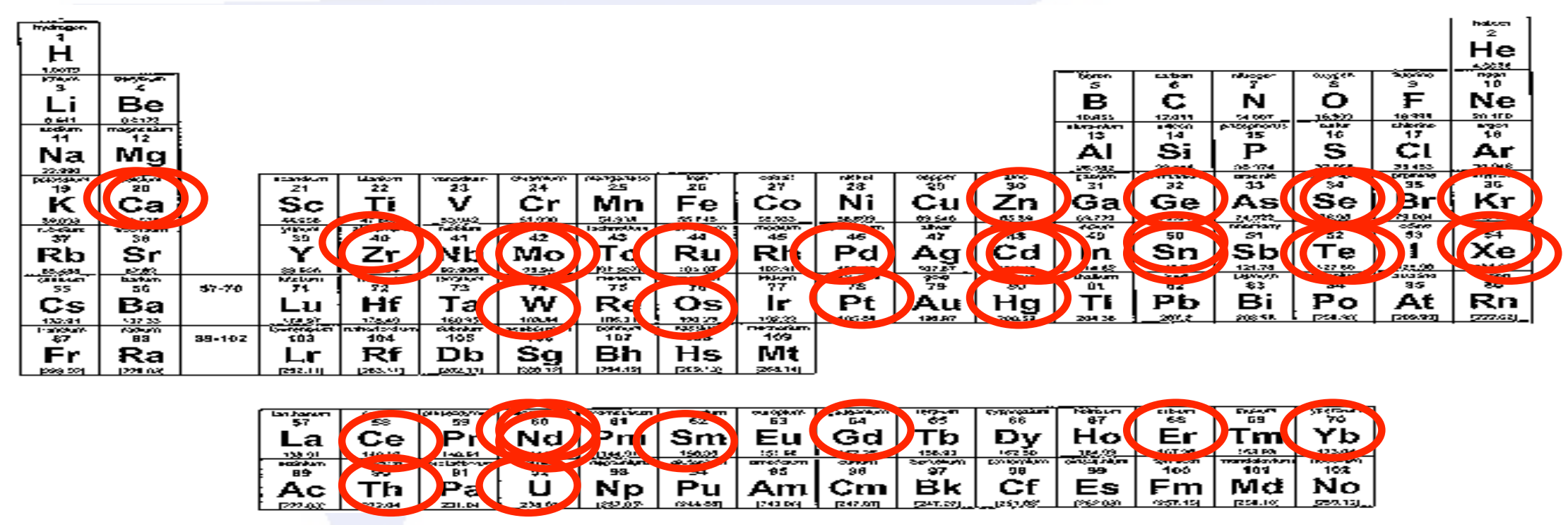




\section{How to choose a $\beta \beta$ isotope?}

- Detector technology exists

- High isotopic abundance or an enriched source exists.

- High energy = fast rate, above background 


\section{$\beta \beta$ Candidates \\ Abundance $>5 \%$, Trans. Energy $>2 \mathrm{MeV}$}

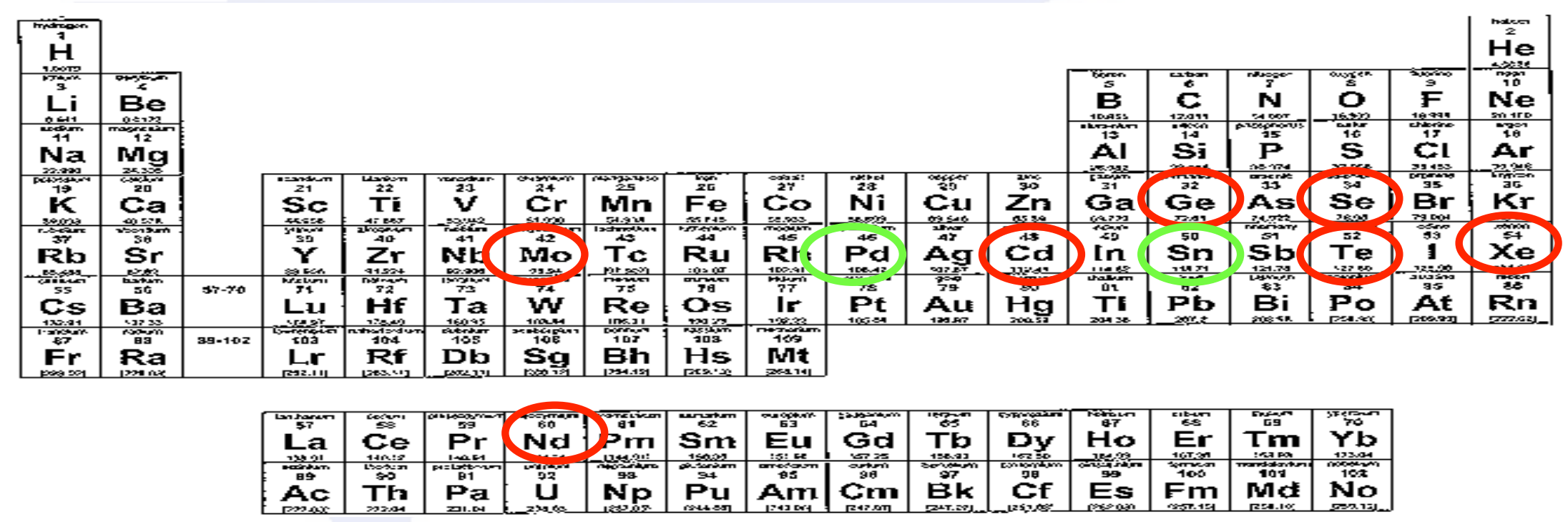

Frequently studied isotope. 


\section{$\beta \beta$ History}

- $\beta \beta(2 v)$ rate first calculated by Maria Goeppert-Mayer in 1935.

- First observed directly in 1987.

- Why so long? Background

$$
\begin{gathered}
\text { - } \tau_{1 / 2}(\mathrm{U}, \mathrm{Th}) \sim \mathrm{T}_{\text {universe }} \\
\text { - } \tau_{1 / 2}(\beta \beta(2 v)) \sim 10^{10} \mathrm{~T}_{\text {universe }}
\end{gathered}
$$

- But next we want to look for a process with:

$$
\text { - } \tau_{1 / 2}(\beta \beta(0 v)) \sim 10^{17} T_{\text {universe }}
$$




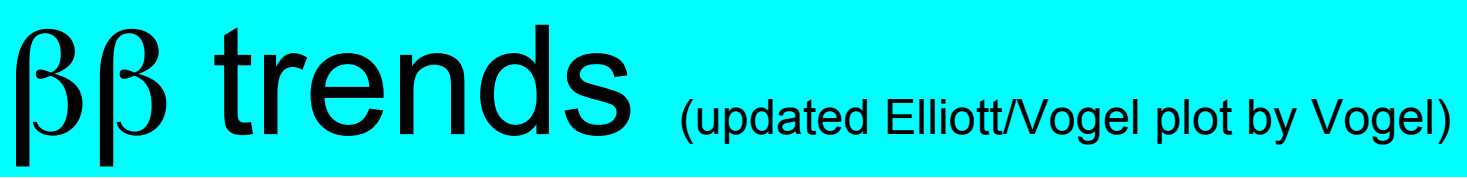

History of the $0 v \beta \beta$ decay

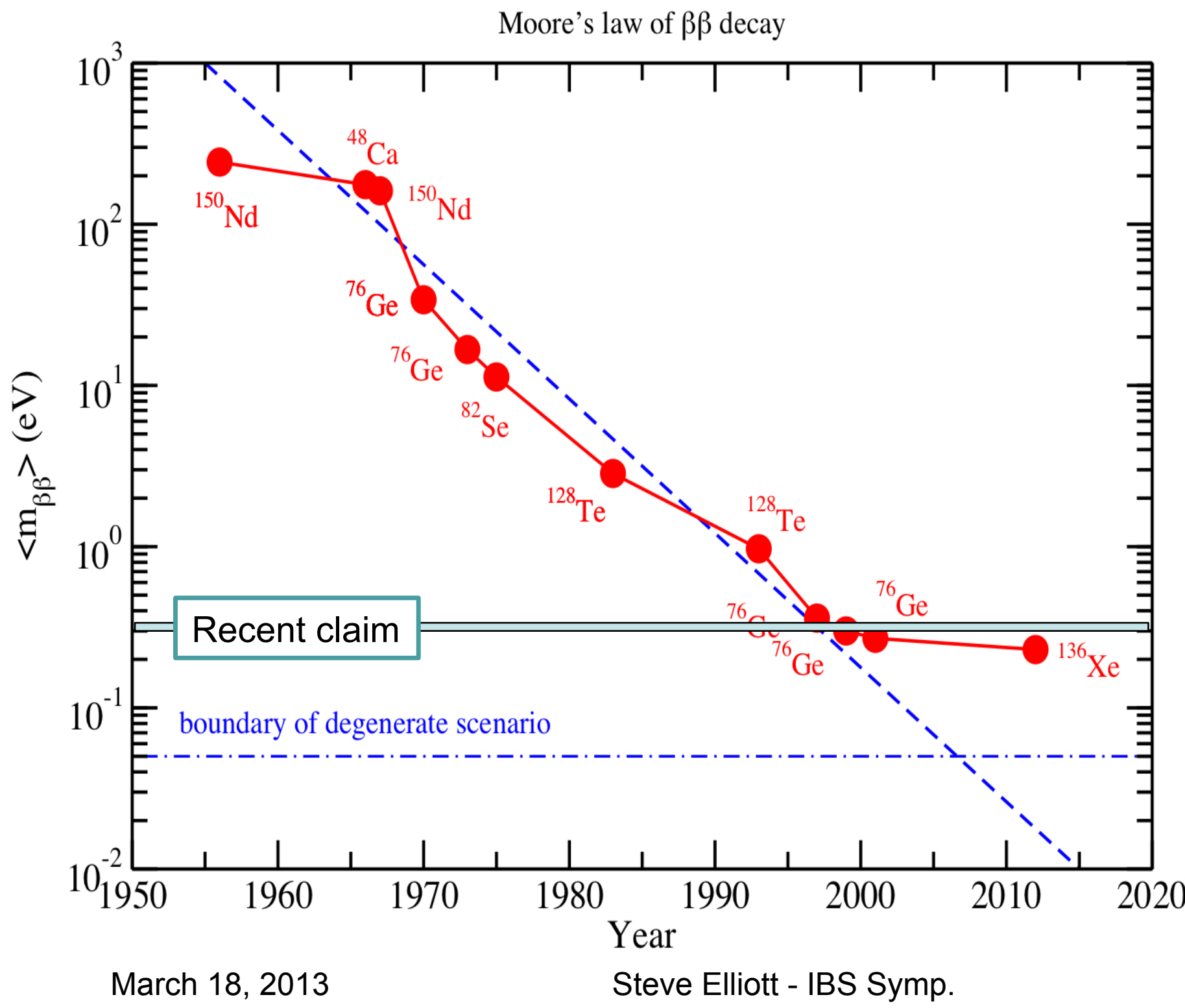

Historically, there are $>100$ experimental limits on $T_{1 / 2}$ of the Ov $\beta \beta$ decay. Here are the records expressed as limits on $\left\langle m_{\beta \beta}\right\rangle$ using one set of nuclear matrix elements (RQRPA of Simkovic et al. 2009.) Note the approximate linear slope vs time on such semilog plot. However, during the last decade the complexity and cost of such experiments increased dramatically. The constant slope is no longer maintained.

March 18, 2013

Steve Elliott - IBS Symp. 


\section{A Claim}

has become a litmus test for future efforts

\section{$\beta \beta$ is the search for a very} rare peak on a continuum of background.

$\sim 70 \mathrm{~kg}$-years of data

13 years

The "feature" at $2039 \mathrm{keV}$ is arguably present.
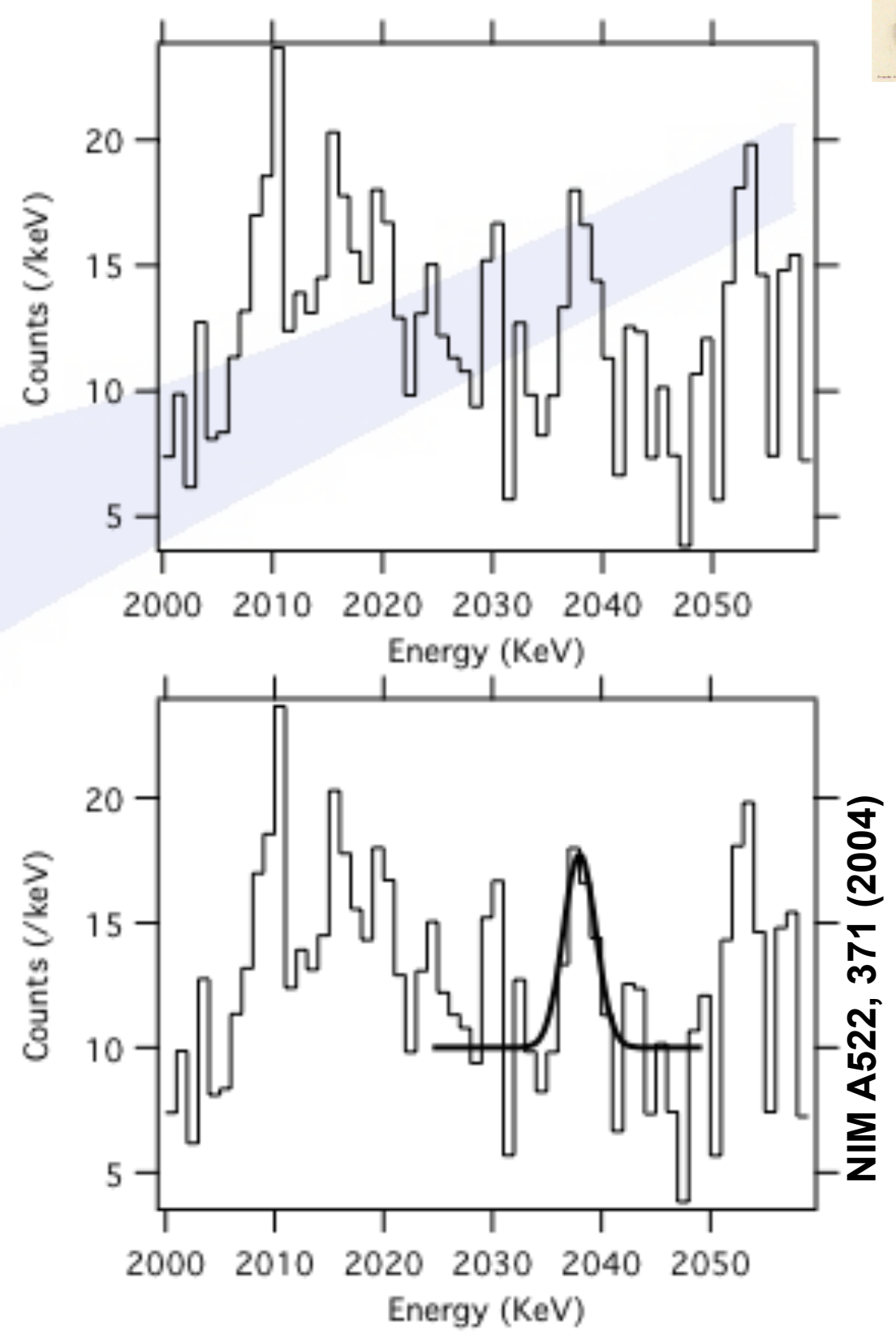


\section{EXO result}

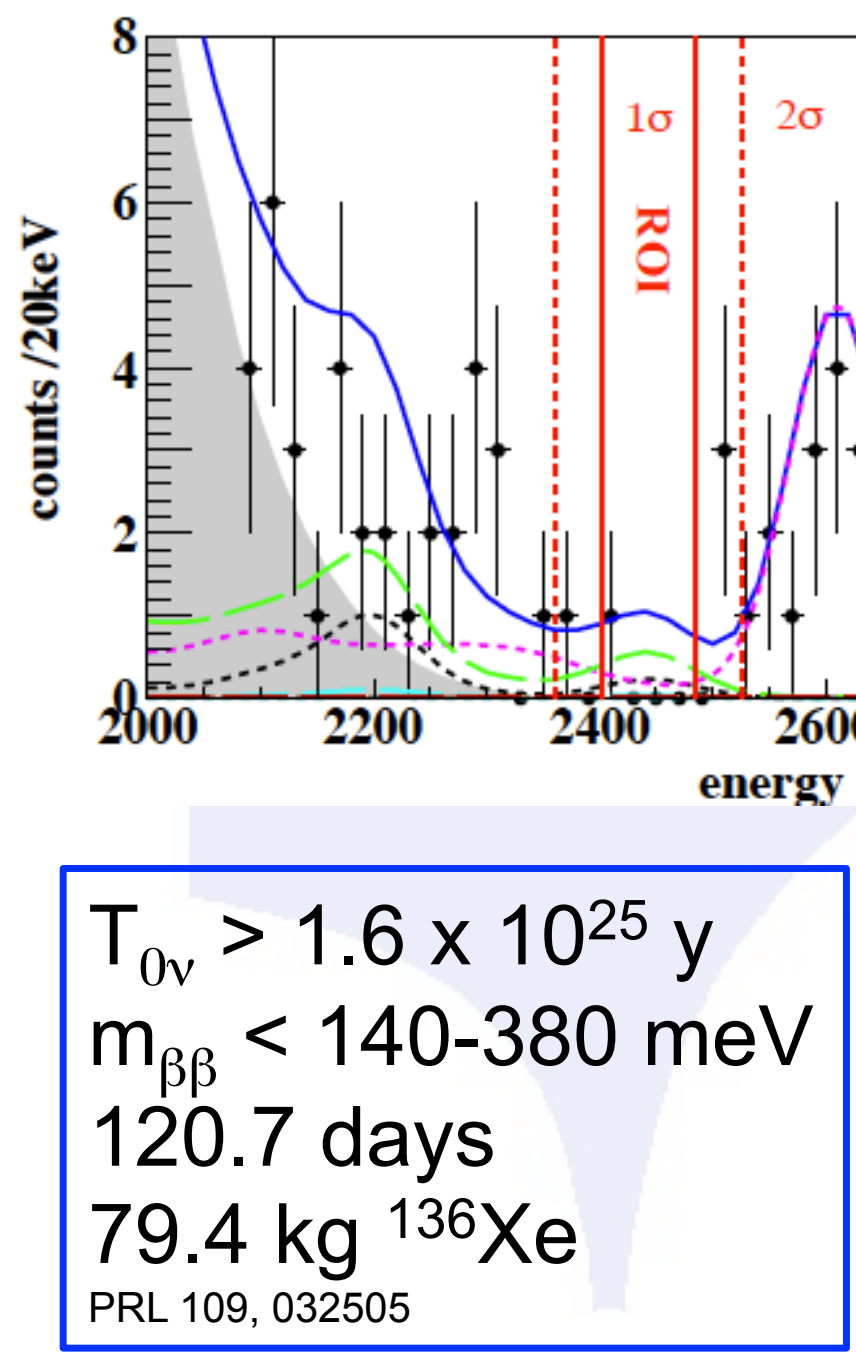

March 18, 2013

\section{SS}

Joint analysis with KamLAND-Zen gives $3.4 \times 10^{25}$ y, $120-250 \mathrm{meV}$ arXiv:1211.3863

\section{Expected events from fit}

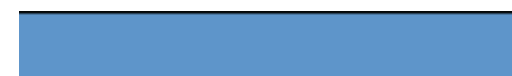

${ }^{222} \mathrm{Rn}$ in cryostat air-gap

${ }^{238} \mathrm{U}$ in LXeVessel

${ }^{232} \mathrm{Th}$ in LXeVessel

${ }^{214} \mathrm{Bi}$ on Cathode

All Others

Total

Observed

Background index b $\left(\mathrm{kg}^{-1} \mathrm{yr}^{-1} \mathrm{keV}^{-1}\right)$

Steve Elliott - IBS Symp. $\pm \mathrm{I} \sigma$

$\pm 2 \sigma$

$\begin{array}{llll}1.9 & \pm 0.2 & 2.9 & \pm 0.3\end{array}$

$\begin{array}{llll}0.9 & \pm 0.2 & 1.3 & \pm 0.3\end{array}$

$\begin{array}{llll}0.9 & \pm 0.1 & 2.9 & \pm 0.3\end{array}$

$\begin{array}{llll}0.2 & \pm 0.01 & 0.3 & \pm 0.02\end{array}$
$\sim 0.2 \quad \sim 0.2$

$\begin{array}{llll}4.1 & \pm 0.3 \quad 7.5 \quad \pm 0.5\end{array}$

I 5

$1.5 \cdot 10^{-3} \pm 0.1 \quad 1.4 \cdot 10^{-3} \pm 0.1$ 


\section{Future Data Requirements}

Why wasn't the claim sufficient to avoid controversy?

- Low statistics of claimed signal - hard to repeat measurement

- Background model uncertainty

- Unidentified lines

- Insufficient auxiliary handles Result needs confirmation or repudiation 


\section{An Ideal Experiment Maximize Rate/Minimize Background}

$\left\langle m_{\beta \beta}\right\rangle \propto\left(\frac{b \Delta E}{M t_{\text {live }}}\right)^{\frac{1}{4}}$

Large Mass ( 1 ton) Large $Q$ value, fast $\beta \beta(0 v)$ Good source radiopurity Demonstrated technology Ease of operation Natural isotope Small volume, source $=$ detector Good energy resolution Slow $\beta \beta(2 v)$ rate Identify daughter in real time Event reconstruction Nuclear theory 


\section{Signal:Background 1:1 Its all about the background}

\begin{tabular}{|c|c|c|}
\hline $\begin{array}{l}\text { Half life } \\
\text { (years) }\end{array}$ & $\begin{array}{c}\text { 〜Signal } \\
\text { (cnts/ton-year) }\end{array}$ & $\begin{array}{c}\text { Neutrino mass } \\
\text { scale }(\mathrm{meV})\end{array}$ \\
\hline $10^{25}$ & 530 & 400 \\
\hline $5 \times 10^{26}$ & 10 & 100 \\
\hline $5 \times 10^{27}$ & $\begin{array}{c}\text { To reach } \\
\text { atmospheric } \\
\text { scale need BG }\end{array}$ & 40 \\
\hline$>10^{29}$ & $<0.05$ & $<10$ \\
\hline
\end{tabular}

Degenerate

Atmospheric

Solar 


\section{Sensitivity, Background and Exposure}

Goal is to achieve ultra-low backgrounds of less than 1 count per ton of material per year in the ROI about the $\beta \beta(0 v) Q$-value energy.

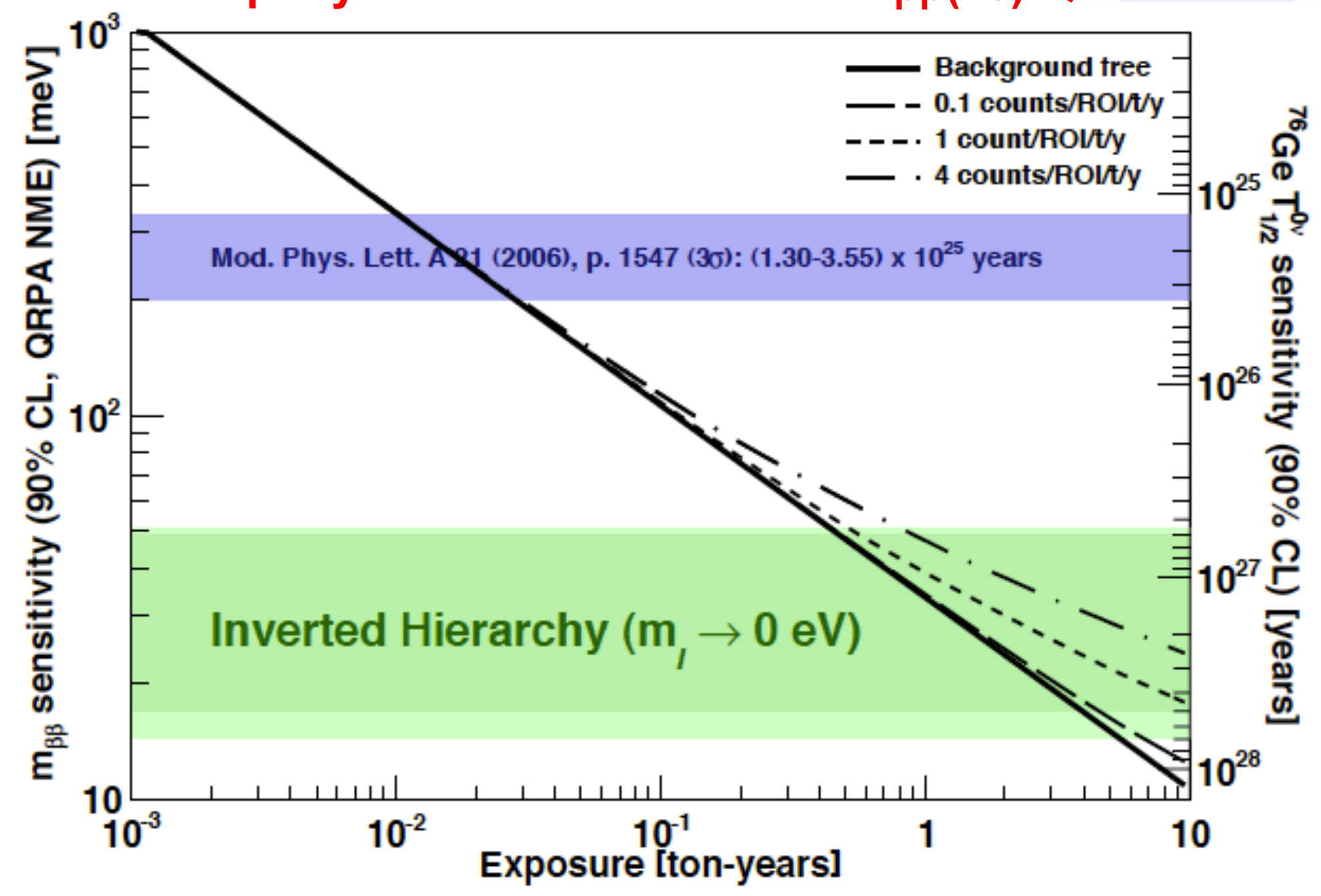




\section{Background in Recent Experiments}
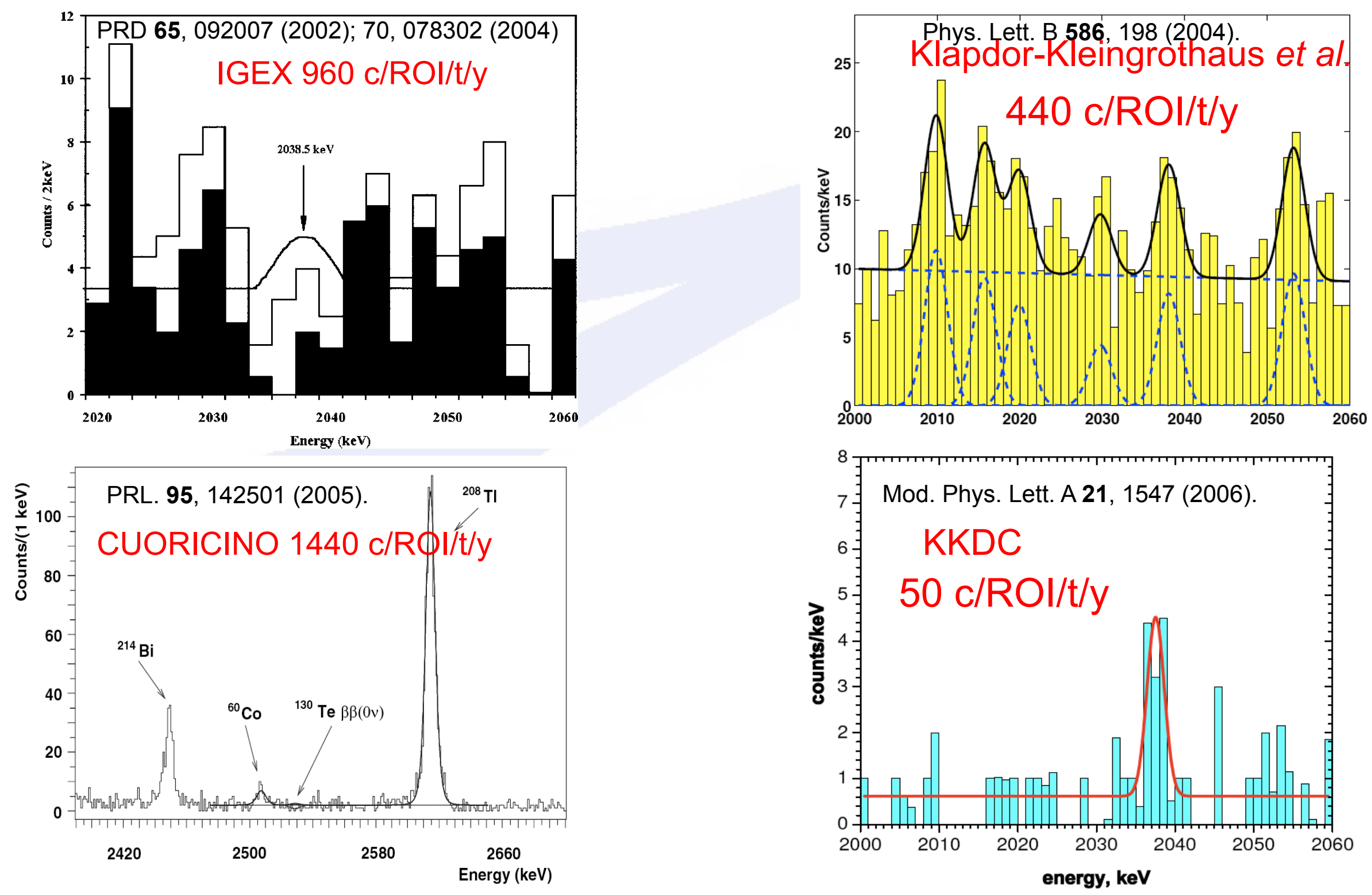

March 18, 2013

Steve Elliott - IBS Symp. 


\section{Background in Recent Experiments}
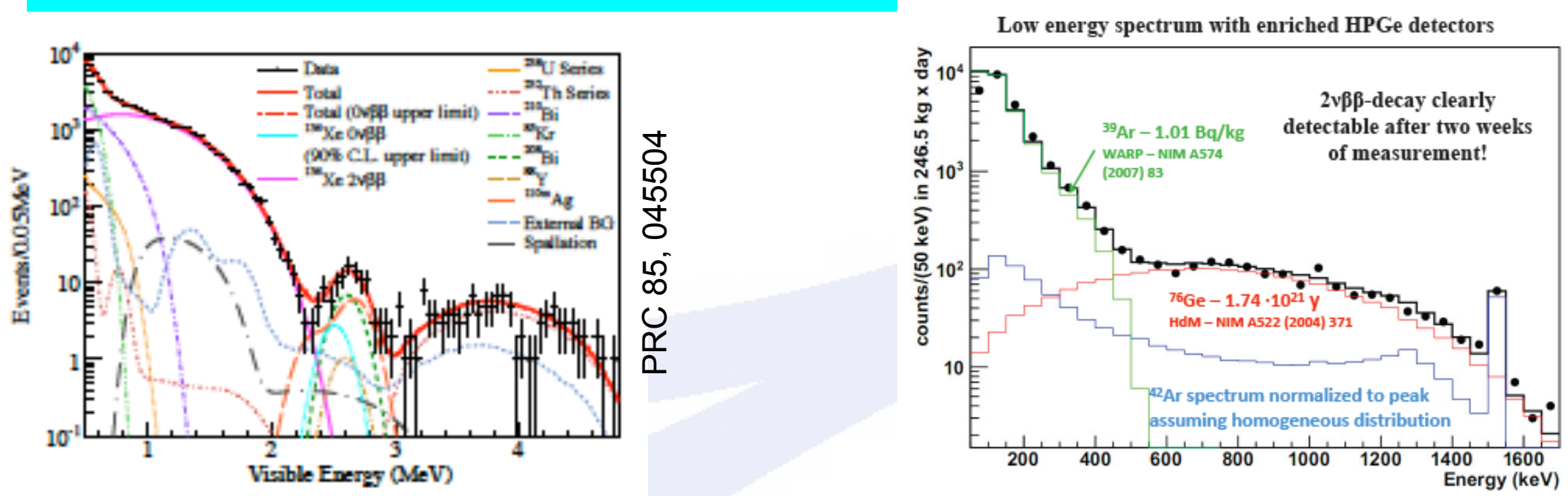

KamLAND-Zen 2400 c/ROI/t(Xe)/y

EXO-200 129 c/ROI/t/y

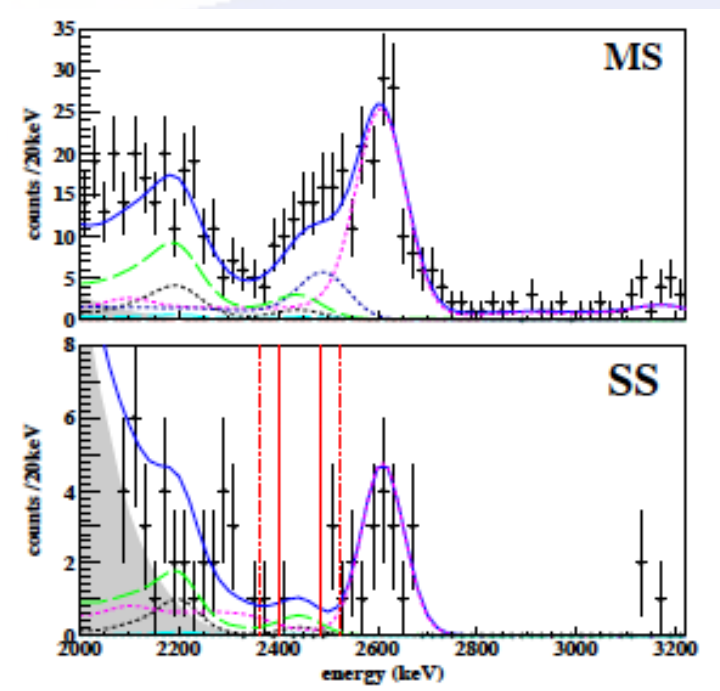

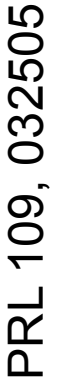

GERDA - init. enr. $81 \mathrm{c} / \mathrm{ROI} / \mathrm{t} / \mathrm{y}$

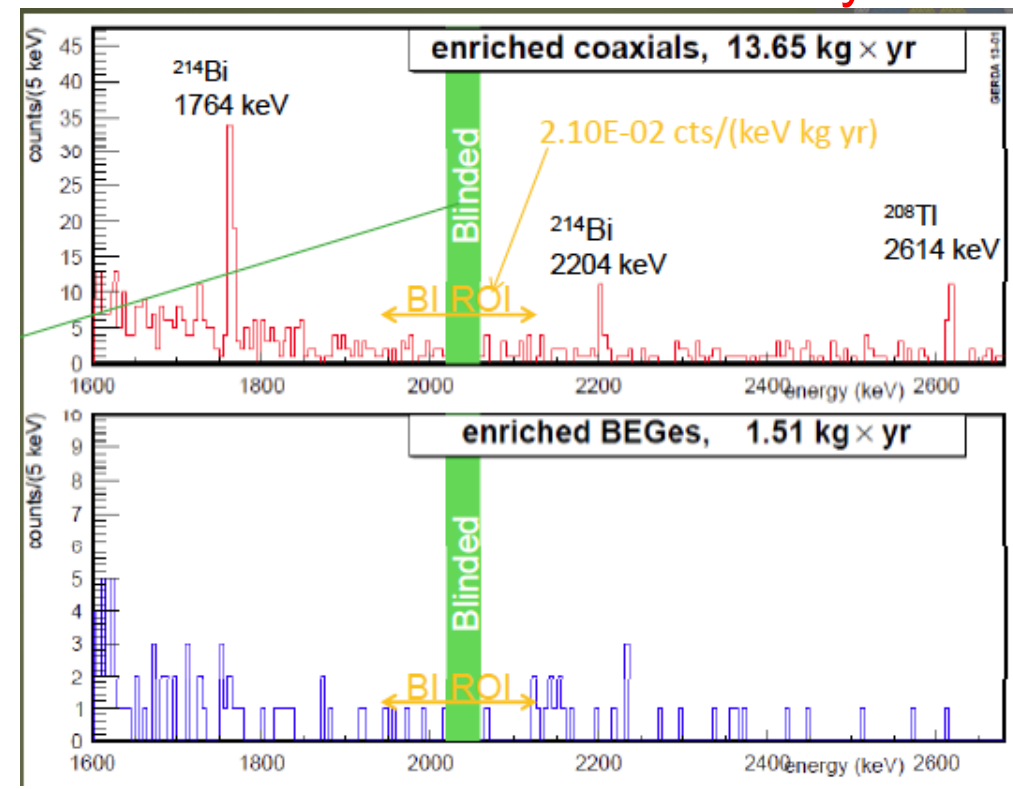

March 18, 2013

Steve Elliott - IBS Symp. 


\section{Previous Background Levels}

Experiment

Background (cnts/ ROI-t-y)

IGEX

Heid-Moscow

CUORICINO

GERDA

EXO-200

KamLAND-Zen
960 (400 with PSD)

440 (50 with PSD)

1440

81 (no PSD)

130

55( 2400per $\mathrm{t}(\mathrm{Xe}))$
Width

(1 FWHM)

$4 \mathrm{keV}$ ROI

$4 \mathrm{keV} \mathrm{ROI}$

$8 \mathrm{keV} \mathrm{ROI}$

$4 \mathrm{keV}$ ROI

$106 \mathrm{keV}$ ROI

(1.8\% 1 sig resol.)

Width not

explicitly given

Background is per tonne of material - big difference for KamLAND-Zen 


\section{Background Considerations}

At atmospheric scale, expect a signal rate on the order of 1 count/tonne-year

- $\beta \beta(2 v)$

- natural occurring radioactive materials

- neutrons

- long-lived cosmogenics 


\section{Great Number of Proposed Experiments}

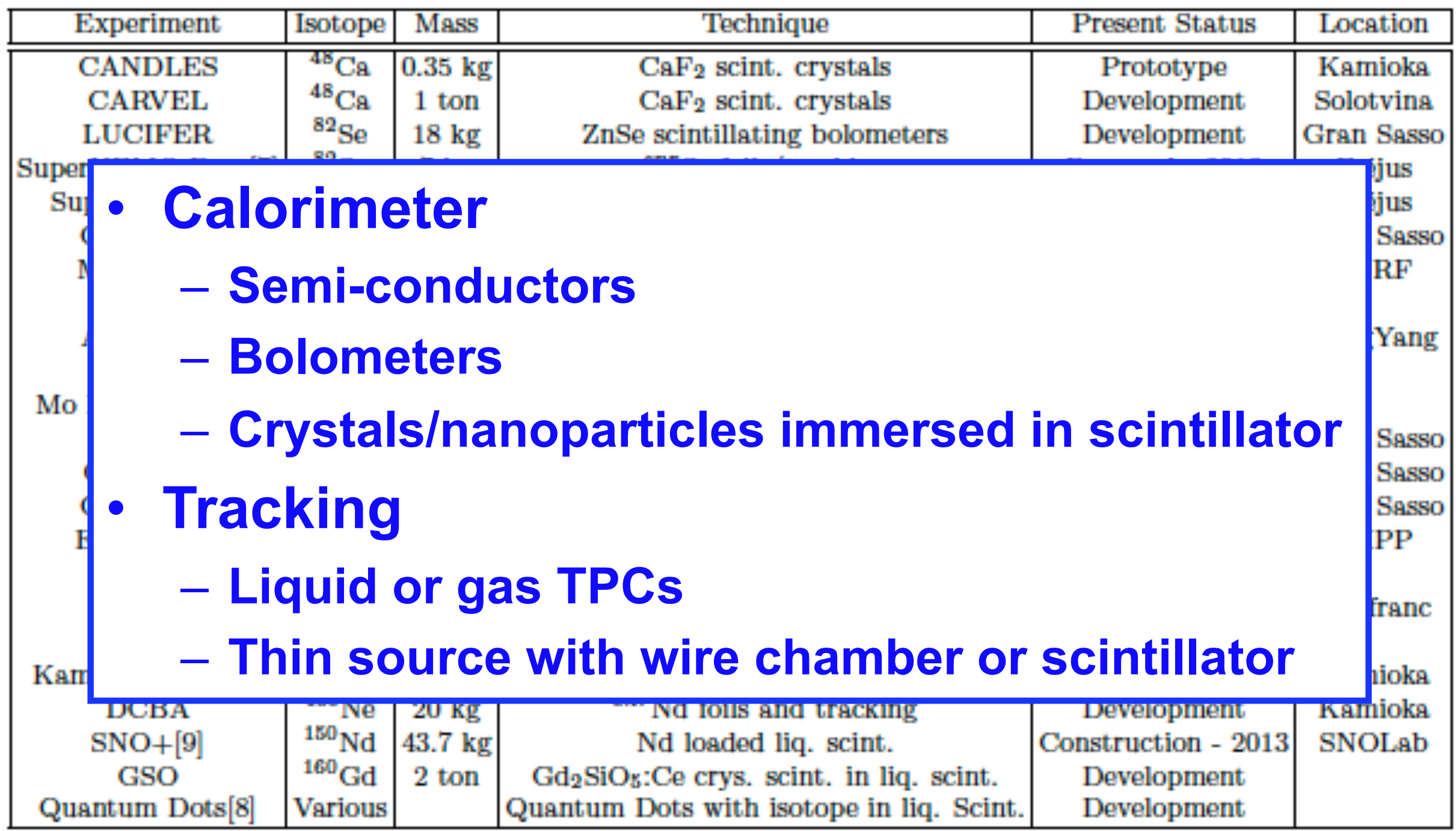


Experiments that will test claim in coming few years.

\begin{tabular}{|l|c|c|}
\hline & Mass & Run Plan \\
\hline CUORE & $\sim 200 \mathrm{~kg}$ & 2014 \\
\hline EXO-200 & $\sim 100 \mathrm{~kg}$ & 2011 \\
\hline GERDA I/II & $\sim 34 \mathbf{~ k g}$ & $2011 / 2013$ \\
\hline KamLAND-Zen & $\sim 125 \mathrm{~kg}$ & 2012 \\
\hline MAJORANA & $\sim 30 \mathrm{~kg}$ & 2013 \\
\hline NEXT & $\sim 100 \mathrm{~kg}$ & 2014 \\
\hline SNO+ & $\sim 44 \mathbf{~ k g}$ & 2014 \\
\hline SuperNEMO Dem. & $\sim 7 \mathbf{~ k g}$ & 2013 \\
\hline
\end{tabular}

Good guess that we'll reach about $100 \mathrm{meV}$ in the 2013-2015 time frame.

Ton-scale projects might be starting by 2020. 


\section{Discovery vs. Measurement a future decision point}

Expt. Size: up to $10 \mathrm{~kg}$ Sensitivity: $\sim 1 \mathrm{eV}$

$\sim 10 \beta \beta(2 v)$ measurements
Expt. Size: 100-200 kg Several experiments Program to measure rate in several isotopes

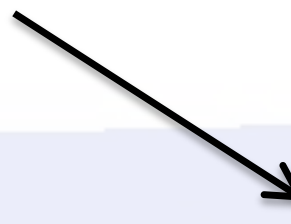

Expt. Size: $30-200 \mathrm{~kg}$ Sensitivity: $100 \mathrm{meV}$ Quasi-degenerate 8-10 expts. worldwide

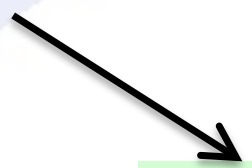

Expt. Size: $\sim 1 T$

$\sim 3$ expts.

Sensitivity: $50 \mathrm{meV}$

Atmos. scale
Expt. Size: few T $>3$ experiments

Program to measure rate in several isotopes Kinematic meas. 


\section{Take-Home Message}

- Due to the minimum neutrino mass scale implied by the neutrino oscillation experiments:

- The next generation $\beta \beta$ experiments have a good possibility of reaching an exciting $\left\langle\mathrm{m}_{\beta \beta}>\right.$ region.

- The MAJORANA DEMONSTRATOR is making strong progress

- Enriched material order

- Detector contract in place

- Lab ready to go

- A large scale experiment will be proposed and R\&D is beginning. 